\title{
Coping with homeschooling and caring for children during the UK COVID-19 lockdown: voices of working mothers
}

Angeliki Kallitsoglou ( $\sim$ angeliki.kallitsoglou@roehampton.ac.uk)

University of Roehampton https://orcid.org/0000-0002-6497-4379

Pamela-Zoe Topalli

University of Turku

\section{Research Article}

Keywords: COVID-19, UK, working mothers, homeschooling, childcare, emotional sates, wellbeing

Posted Date: March 16th, 2021

DOl: https://doi.org/10.21203/rs.3.rs-333649/v1

License: (c) (1) This work is licensed under a Creative Commons Attribution 4.0 International License.

Read Full License 


\section{Abstract}

We examined working mothers' experiences and feelings about homeschooling and caring for children while working during the summer 2020 COVID-19 lockdown in the UK. Eligible mothers were invited to participating in an on-line survey of open-ended questions that was distributed via social media between mid - June to mid-August 2020. Participants ( $n=47 ; M_{\text {age }}=39.6$, range $=28$ to 54 years) were predominantly white married working mothers and most had a higher education degree. The experience of combining homeschooling/caring while working was perceived difficult by many mothers and it was associated with feelings of stress about managing competing demands concurrently, guilt for not meeting the child's needs, and worry over child socioemotional well-being and academic learning. Support from partner, work, and school were instrumental in improving maternal experience and emotional state. Finally, coping strategies that reflect mindfulness practices for stress management, were associated with positive experiences and feelings.

\section{Background}

In March 2020, quarantine type measures including school and day care centre closures to mitigate the impact of the novel coronavirus (COVID-19) on public health made the combination of home-schooling and/or caring for children while working the 'new normal'. In Great Britain, between April to June 2020, $87 \%$ of caregivers were home-schooling their children with mixed results as, for instance, half $(52 \%)$ of caregivers with school-aged children reported difficulties continuing home - education (Williams et al., 2020).

As well as serious psychosocial effects on children (Holmes et al., 2020; Morgül et al., 2020; Spinelli et al., 2020), closures of schools and childcare settings affect family wellbeing, with mothers being more severely impacted. Mothers undertook the lion's share in home-schooling and caring responsibilities during the lockdown. Research from the Office for National Statistics (ONS) in the UK showed that men and women with a school-aged child (5-18 years) spent similar amounts of time on homeschooling (Williams et al., 2020). However, overall women spent more time on both developmental and non-developmental childcare, such as reading a book and dressing, respectively, with time spent on non-developmental childcare exceeding that of men by $77 \%$ (Office for National Statistics, 2020). Moreover, a large survey from the Institute of Fiscal Studies, UK showed that mothers were likely to combine paid work with other activities, almost always childcare, in $47 \%$ of their work hours, compared with $30 \%$ of fathers' work hours (Andrew et al., 2020b) .

Previous research has shown that gendered division of home labour is often related to poor maternal mental health and wellbeing including feelings of unfairness (Lachance-Grzela et al., 2019), more housework and childcare -related multitasking resulting in negative emotions, psychological distress, and work-family conflict (Offer \& Schneider, 2011), and increased mental load over concerns about domestic issues such as childcare, housework, and homeschooling (Daminger, 2019). A large study of the association between biomarkers of chronic stress and flexible working arrangements found that women 
who worked 37 hours or more and had childcare responsibilities were at a significantly higher risk for chronic stress than mothers who worked fewer hours and men showing that the demands of combining full time work and looking after children are particularly pressing (Chandola et al., 2019). Moreover, earlier research on the association between homeschooling and emotional burnout in mothers suggests that home education was taxing on maternal wellbeing even before the lockdown (Lois, 2006).

In addition to important consequences on maternal participation in paid work and economic security (Czymara et al., 2020), the unequal division of home schooling and caring responsibilities during the lockdown is supposed to have impacted on maternal wellbeing. In Great Britain, of all the caregivers who were homeschooling during the 2020 lockdown, one in three women (34\%) felt that it was negatively affecting their well-being compared with one in five men (20\%) (Williams et al., 2020). Findings from a large survey in Germany suggested that mothers experienced heavier mental load about domestic responsibilities compared to fathers during the 2020 lockdown (Czymara et al., 2020). Additionally, the combination of paid work and childcare during the 2020 lockdown was associated with emotional distress, such as sadness and frustration, and a negative impact on daily routines, such as poor sleep quality, in a sample of mother in Italy (Di Giorgio et al., 2020). The psychological impacts are expected to be more dramatic for lone-mother households because of additional strain on overstretched emotional and/or financial reserves (Arnot, 2020). The loss of formal and informal sources of childcare and associated unsupported juggling of domestic, childcare and/or work responsibilities is likely to increase the risk of financial insecurity and poor psychological wellbeing among lone-mothers (Arnot, 2020; Mantouvalou, 2020).

The recognition of the pressures from gendered division of unpaid labour on maternal wellbeing is an important equity and public health imperative. Perceived inequality and associated feelings of unfairness and psychological distress can often put mothers at risk of poor mental health (Lively et al., 2010). Because maternal health and wellbeing is a key determinant of child physical and mental health (Cooper \& Stewart, 2013; Leijdesdorff et al., 2017), the distress caused by the consequences of the unequal division of labour may be inadvertently impacting on children's outcomes. Furthermore, acknowledging the differential influence of the outbreak on women and men is a fundamental step towards understading the impact of the state of health emergency, and for developing effective and equitable policies and interventions (Wenham et al., 2020). As a result, the research community and international and national organisations are endorsing the need for women's experiences and knowledge inclusion in the decision making for pandemic crisis management and post-COVID-19 recovery measures (Power, 2020; Wenham et al., 2020).

As many countries are implementing further universal lockdown measures including short-term school closures to mitigate the continuous impact of the pandemic, it is necessary that we give voice to those experiences to help identify efforts to mitigate its secondary effects on maternal wellbeing. To date, we know that mothers have been adversely influenced by having taken up the lion's share in unpaid domestic and childcare work during the 2020 lockdown. However, we know very little about the details of their experiences and associated feelings and challenges that will help inform the development of meaningful 
policies and social innovations. To contribute to the global efforts of giving voice to those experiences we extend this work by asking mothers to share them as well as their feelings and thoughts about the challenges of homeschooling and/or caring for children while working during the COVID-19 2020 lockdown.

\section{Methods}

\section{Participants and procedures}

Webropol (https://webropol.com/) was used to develop an electronic survey to capture maternal experiences of working while homeschooling and/or caring for children and the family during the lockdown as part of a larger project on maternal mood during the COVID-19 lockdown in the summer and spring of 2020. In the UK, the questionnaire was distributed between mid of June and first week of August 2020 via social media, such as mother groups on Facebook, our personal networks, and social networking platforms such as Linkedln. At the time, the UK government was imposing important social distancing so not everyone had returned to school and not all parents had opted for summer camps. Ethical approval was granted by the Ethics Committee for Human Sciences at the University of Turku, Humanities and Social Sciences Division.

Fifty-five mothers consented to complete the questionnaire. The data of those who were not residing in the UK during the lockdown $(n=1)$, who were not working $(n=5)$, did not provide evidence of whether they were working $(n=1)$ and those with incomplete questions $(n=1)$ were not included in the study. The mothers in the final sample $(n=47)$ were primarily white $(81 \%)$ working mothers ( $53 \%$ full-time) between 28 and 54 years old $\left(M_{\text {age }}=39.6 S D=5.65\right)$. Children were between 1 to 17 years old and $24(57 \%)$ households had more than one child. Most mothers (81\%) were married and had at least a higher education degree (85\%). In 2019, $14.9 \%$ of families in the UK were lone parent families and of those with dependent children $22.3 \%$ were lone families (Office for National Statistics, 2019), therefore our sample had a good representation of single parent households $(n=9(19 \%))$.

\section{Measures}

Topalli and colleagues (Topalli et al., 2020) developed a questionnaire which comprised 10 questions on maternal demographics and 6 open-ended questions designed to capture how mothers coped emotionally and practically with the challenges of the lockdown on the following domains: 1) general mood, thoughts and feelings, 2) working life (e.g., mode of work, changes in workload, etc.); 3) social life (e.g., interactions with friends, social activities, etc.); 4) daily routines (e.g., daily routines including meal times, sleeping quality, and sleeping schedule, physical activities/exercising, leisure time, etc.); 5) combining work with childcare, homeschooling, and homecare, 6) views and attitudes about opening up the society /returning to normal. 
We used thematic analysis which allows identifying, analysing, and reporting patterns (themes) within data (Braun \& Clarke, 2006, p. 79). A theme refers to pieces of information that are relevant to the research question and represents some level of patterned response or meaning within the data set (Braun \& Clarke, 2006, p. 20). Participant responses which are linked under a common idea are assigned codes which are then grouped in themes based on whether they cluster together. because we did not use categories. The questions were semi-structured and guided the participants' answers, but we did not use them as an a priori analytical framework. Therefore, the type of thematic analysis we used was inductive.

We used a 6 -step guide of conducting thematic analysis described in Braun and Clarke (2006): familiarising with the data; initial code generation; theme development; theme review; theme definition; report production. Briefly, we read participants' answers carefully, including answers to the questions that were designed to explore maternal experiences in domains other than managing childcare and/or homeschooling while working (i.e. questions $1,2,3,4 \& 6$ ), to identify meaningful units of text that were relevant to the topic. Then, units of text dealing with the same topic were grouped together to form categories that were given provisional definitions. The same unit of text could be included in more than one category. We then reviewed the categories to ensure that the data identified for each one was exhaustive and that they reflected the definition of the category that they were grouped under. The analysis resulted in nineteen categories which were grouped into six key themes: the experience of homeschooling and/or childcare, maternal emotional states, coping strategies, support, delivering and managing homeschooling, and child wellbeing and academic achievement.

Primary coding was performed by the first author using the NVivo 12 software for qualitative data analysis. First, question 5 was coded followed by the remaining questions for units of extract relevant to the topic. Question 1 about general emotional wellbeing and question 2 about how work and/or study impacted on mood and feelings provided some mothers with the opportunity to elaborate on concerns about childcare and/or homeschooling and how work had impacted on their capacity to juggle. No relevant information was identified in the social life (3), daily routines (4), society open - up (6) questions.

To examine reliability of themes identified by the first author the second author coded all the data. First, a discussion took place on how to approach the coding as the authors did not rely on a priori analytical frameworks nor did they use the questions as a framework, but instead attempted to allow the data to drive the coding. The second author identified four themes that closely matched those identified by the first author: maternal emotional state, coping strategies, managing challenges with homeschooling and/or childcare which was similar to the experience of homeschooling and/or childcare theme identified by the first author, and challenges with home-schooling. A discussion between the two coders led to the following theme reduction decisions. The categories of the support theme identified by the first author were instead diffused across the different themes identified by the second author, but because of its prominence it was decided to keep it as a theme of its own. The themes relevant to the challenges of delivering and managing homeschooling identified by both authors was incorporated into the experience of homeschooling and/or childcare theme because it was instrumental in shaping maternal experiences. 
Finally, the theme child wellbeing and academic achievement identified by the first author was initially included by the second author as a category of the theme managing challenges with homeschooling and/or childcare. Because the common thread in this theme was worry about children's wellbeing and achievement it was incorporated in the emotional states theme. The final four themes were: the experience of homeschooling and/or childcare, maternal emotional states, support, and coping strategies.

\section{Findings}

The experience of homeschooling and/or childcare while working during the lockdown

To enhance the interpretation of the data, first, we run a word frequency analysis, included in the NVivo stemmed word function, to isolate the adjectives and adverbs that mothers used to describe their experiences. The most common words were difficult $(n=10)$, followed by hard $(n=5)$, stressful $(n=4)$, struggle $(n=4)$, challenging $(n=3)$, frustrating $(n=3)$, impossible $(n=2)$, and tough $(n=2)$.

Further analysis of the extracts that reflected maternal experiences reveal how their daily realities had changed because of having to be present for their children as well as at work. The experience of the mother below reflects the experience of many mothers in the sample:

I have been working normal hours and had my usual caseload. On top of that I had to look after my son, so often I would be in a session and at the same time had to make sure my son doesn't climb on the sofa and falls down!

The experience of homeschooling itself was challenging too as it was an add-on to an already heavy daily routine and a cause of physical and mental exhaustion

I've always had to juggle working full time and looking after the children [....] what compounded my feelings of frustration has been the home schooling

What seemed to be the most challenging aspect of it was synchronous delivery which involved having to manage conflicting demands, while trying to handle constant interruptions, and unforeseeable events such as child emotional outburst and sibling fights.

(i feel) super stressed as I have had for the most part of it very broken time between teaching kids and working and getting working time interrupted all the time

I have been trying to deliver homeschooling in set periods so I can have set times I am at my desk but that has not always been feasible as I have been managing disagreements between my eldest two children.

This was intensified by child age, special educational needs (SEN) and temperament. For instance, young children needed more support to engage with homework or activities, SEN provision was not always easy 
to be implemented remotely, and child reluctance to engage with homework. Moreover, lack of motivation to engage with home learning was reported by many mothers. Finally, lack of access to electronic devices, and confidence with teaching were important determinants of the experience.

I am not a teacher, my daughter wants me to be her Mum and not her teacher - this has been learnt the hard way through upset and tears and tantrums-hers and mine!

A few mothers shared more positive experiences pointing out to some of the factors that made the experience less challenging such as less pressure from work, children's desire to be homeschooled, managed to be organised, and working-remotely and associated benefits such as saving time because of not having to travel to work. However, there were other spheres of life that suffered, such as mental health and work.

Remote working is great for time managing and cutting out travel time but causes anxiety because you are not that visible at work.

When we looked at the data from the point of view of single mothers $(n=9)$ all but one agreed that it was difficult to manage competing demands. Homeschooling was reported as the most challenging aspect of the lockdown and the cause of losses at work. Additionally, the intersection of the extra pressure of juggling competing responsibilities alone and factors pertaining to the mental and emotional load caused by additional carrying responsibilities, for instance cohabiting elderly parents, and/or by conflict between ex-spouses may put lone mothers at a greater risk of poor wellbeing.

I was helping both with school work till just before summer half term but my work suffered and I lost a client. It has been a terrible situation made worse by stresses of living with elderly parents [...].

\section{Emotional states}

First, we run a word frequency analysis using the stemmed word function on a list of isolated emotions after excluding emotional states mentioned twice from the same individual. After amending for words that were not clustered together because of typos or not grouped by the software (e.g., guilt/guilty) we found that mothers experienced a wide range of negative feelings with the most common being feeling stressed $(n=9)$, guilty $(n=8)$ and worried $(n=4)$ followed by frustrated, pressured, and tired $(n=3)$, angry, conflicted, disappointed, failed, proud, and resentful $(n=2)$.

Then, we coded all the extracts that reflected emotional states including those reported more than once but appearing in a different context, for instance resentful towards husband and to parents eligible to send their kids to school, to capture the whole spectrum of triggers of maternal emotional responses to the situation. The most common reason reported for feeling stressed was having to combine and deliver competing tasks such as work, homeschooling/caring for children, and household chores.

I was feeling rather stressed about combining both work duties, household and homeschooling. 
Lack of time, setting-up a routine for both parent and child, constantly being interrupted, and homeschooling were a few additional reasons for feeling stressed.

Feelings of guilt/feeling guilty varied from not contributing as much as the husband to children's homeschooling, to not being good enough, not spending enough time/making an effort to homeschool and support children's learning, and not measuring up to other parents. Additionally, a few mothers made links with the pressure from having to deal with competing demands and associated conflict and guilt that come with it when they are prioritised over the parenting/caring role.

I feel guilty when I am taking care of my children that I am not working or keeping up with housework and when I work I feel guilty that my kids are watching too much TV.

A lot of mothers were deeply worried over the impact of the lockdown on child wellbeing and academic achievement. Work-related expectations and pressure to deliver, triggered for some a chain of emotional reactions which were expressed as stress, family conflict, poor parenting behaviour and associated feelings of guilt, worry and inadequacy:

I have felt incompetent many times and stressed about keeping a routine for my son and do my job. I have also felt guilty for not meeting my son's needs as I would expect so and also feeling like I am falling behind in terms of the quality of service I offer [...]. All this pressure often manifests in arguments with my husband but also shouting at our son when we feel we have had enough which is something against our general parenting style. This of course creates more guilt and feelings of letting our son down but also worry about his development.

When we reduced the list of feelings by grouping them in to clusters reflecting similar underlying emotional states stress and guilt were still the most prevalent, but anger and resentment (i.e. angry, upset, resentful, annoyed) because of unequal distribution of homeschooling/childcaring/household chores and not being able to send kids at school like other parents, as well as feelings of failure and incompetence (i.e. failed, incompetent, not good enough, not coping, rubbish, can't do it all) were common. Additionally, feelings that reflect physical and mental exhaustion (i.e., tired, drained, exhausted), unfairness, and boredom and monotony were also common.

Another important cluster of feelings that potentially allude to symptoms of anxiety and low mood emerged. Comments from ten mothers that fell under this category include feeling anxious/nervous, panicked, trapped, overwhelmed, low and lonely, and not able to relax/switch off.

Generally, the variety and intensity of feelings did not seem to vary significantly across single mothers and mothers in partnerships. Notwithstanding, the experience of not having anyone to turn to may be particularly challenging to deal with and exacerbate maternal emotional state.

The bad days were feelings of not coping, guilt for not being able to sit with my child and get through the work at his pace, overall guilt that nothing was being done properly because there was too much to do, 
feeling utterly exhausted and having no time to myself (not doing work or not tending to my child's needs) and not being able to ask anyone for help.

Several positive feelings were also reported by a group of mothers which included emotional states that suggest a positive outlook such as staying positive and strong, feeling blessed, creative, motivated and energised although for some energy and motivation waned after a while, and be grateful and patient. Of interest are the feeling of pride and agency that some mothers experienced despite the difficulties.

I have moments of really enjoying the time with my children and pride at managing it all.

\section{Support}

The theme of support - or lack of it - from partner and work was brought up by many mothers. While a few mothers mentioned that their partners took on more responsibilities, most of them reported a lack of support. Gendered distribution of responsibilities was the most reported reason which for some leD to tension over whose work was more important. Other reasons included prioritising partnern's work, partners' increased workload, and disagreements in child upbringing.

... (the lockdown) brought to light that the female is expected to take care of children more than men.

... my husband can 'go to work' in the spare room everyday [... ] and be undisturbed whereas I am always multitasking.

The single mothers in our sample reported receiving no support from their ex - husband. For some the disagreements about children's upbringing or interaction was a source of significant stress and anxiety.

A few mothers reported that their workload and/or the work expectations decreased during the lockdown with employers being supportive, tolerant, and understanding. Remote working saved time from travelling, although not being physically present at work came with worries about being less visible. A few mothers who felt there was little support mentioned increased workload and expectations to be always available which for some led to financial losses as a result of having to take unpaid leave to cope.

Workload added by those who don't have kids has increased the stress levels and constant meetings about things which could be shared over an email instead.

as there is an expectation that we are all constantly available while at work

The school was another important source of support and provision of devices and individualised support for pupil with SEN were welcomed by a few parents. However, others mentioned receiving little or no support from teachers, excessive homework and lack of communication.

find it frustrated, teachers put too much homework, without thinking about parents who are working, is not my job to teach and I had to go and read things before I explained them to my son. I have to work and help him with his homework at the same time 
The on-line lessons were not proven very helpful for everyone.

The daily Google classroom stream became quite oppressive - the 'need' to post delightful pictures of all the rich and exciting activities one was supposed to be doing was irritating. It was quickly dominated by the parents who weren't working and those who were found it hard to engage (me included). That made it harder to handle

Other sources of support included family members such as older children or relatives and neighbours. Support from social media reported in the statements of two mothers suggest that they intensified the anxiety and pressure that mothers experienced rather than offering practical support.

I've found the constant stream of Facebook ideas of 'how to home school your kids' totally frustrating and at times I have felt very guilty that I am not spending the effort and energy that these other parents are home educating their kids and home made experiments etc etc...

Coping mechanisms

Another major theme that appeared in the data was that of mothers' coping mechanisms which reflected strategies that helped moderate the stress of daily life: adopting a realistic approach, having a positive mentality, and self -management and organisation.

A realistic approach involved looking at life as it is and deal with it in a practical manner which for many entailed lowering expectations in terms of keeping the house clean, time spent with kids, work performance, amount of schoolwork completion, and sticking to rigid schedule such as bedtime and screen time.

we've gradually let certain things slip and that's removed some of the stress. Does it matter if the kids aren't studying by 9am? Does it matte(r) if the floors haven't been washed for a week, does it matter if the kids miss showers or baths on some days. Some trade-offs I guess. I did start to intensify become adverse to lockdown motivators, I didn't have free time to learn something new or make my house sparkle, but now I realise it was people just getting by!

but decided that letting them have more screen time was the only way to survive and if we get an hour of school work done with each of them everyday that's a good day - less pressure to do everything well as it's impossible to do everything.

Adopting a positive mentality involved living in the moment and focusing on the positive aspects of the situation such as being grateful for own and family health, for more family time and bonding with children.

My thoughts and feelings were that we should be grateful that we are healthy and well and we should be patient until all this comes to an end. 
For a few, efforts for self-organisation and self-management such as planning activities to keep the kid(s) busy while working and setting a schedule of childcare duties to share between partners allowed to work more efficiently. Finally, very few mentioned that having access to outside space such as having a garden or being close to the countryside or communicating with other parents who shared similar concerns and struggles were helpful.

\section{Discussion}

The break of the COVID-19 pandemic in the Spring of 2020 found caregivers in many countries around the world caring and home-schooling for their children while working. Mothers shouldered most of the extra unpaid workload, but we have not fully understood how this has impact on maternal wellbeing and family life. Our study is one of the few to approach mothers and provide them with the space to share their experience and feelings while working, caring and homeschooling their children during the summer 2020 lockdown in the UK.

One of the key findings was that the experience of having to care and/or home-school while working was perceived difficult, stressful, and challenging by many mothers. One of the most prominent challenges reported was having to execute simultaneously the responsibilities of three competing roles, that of the professional, mother, and teacher, and battling which role to prioritise. This was compounded by certain family characteristics including lack of infrastructure to support home learning, such as available devices and network quality, low confidence supporting homeschooling, low paternal involvement, lack of a support system, partner conflict, and additional caring responsibilities such as elder parents. Additionally, child characteristics such as SEN, age, and receptiveness to home learning influenced the level of perceived difficulty. Other than the associated stress some mothers reduced expectations or gave up home-schooling altogether to protect theirs and their family wellbeing. Research by the Institute for Fiscal Studies, UK has warned about how varying degrees and quality of access to home learning are deepening inequalities (Andrew et al., 2020c) and call for research in to understanding the factors implicated. Our findings revealed some of the factors which could be included in future investigations on the impact of caring for children and home learning while working on children and families and intervention design.

As well as revealing maternal experiences, our study unearthed the associated emotional load. Stress because of the difficulty to juggle competing demands while working was one of the most reported emotional states. Additionally, a lot of mothers felt guilty for failing to attend to the developmental needs of their children and for not being a good enough mother because of having to prioritise work over childcare and/or homeschooling. Worry over the impact of unavailability, low involvement in children's learning, poor parenting behaviour, and lack of stimulation on child wellbeing and academic achievement was another very common feeling. Significant anxiety was experienced by mothers with children with SEN because of the delays in the implementation of their children's provision as well as the uncertainty and associated worry about what the future holds in the post-COVID-19 era. Additionally, stress over not having no one to turn to was reported by single mothers. Our findings confirm earlier contentions that the challenge of balancing paid work and childcare may have negative impacts on maternal wellbeing and 
quality of children's time (Andrew et al., 2020a). Persistent and consistent feelings of stress, anxiety and worry are indicators of poor mental health and guilt is common in mothers who report emotional and physical burnout (Hubert \& Aujoulat, 2018; Sánchez-Rodríguez et al., 2019). Moreover, feelings of inadequacy, worries over not fulfilling one's parental duties and the internal conflict about prioritising work over the parenting role are relevant to the societal pressures to be the perfect parent while manging the household, keeping a paid job, and pursuing career aspirations (Meeussen \& Van Laar, 2018). Pressures from unrealistic expectations put mothers at risk of mental health difficulties (Henderson et al., 2016; Lamar et al., 2019), and jeopardise their career ambitions (Meeussen \& Van Laar, 2018).

Perceived lack of support from partner, work, and school comprised some of the most important reported challenges that exacerbated maternal negative experiences and associated emotions. Our findings showed that a gendered approach to domestic work allocation, including childcare/ homeschooling, was not always by choice and left many mothers feeling emotionally distressed, physically exhausted, and resentful. On the contrary, where mothers reported equitable paternal involvement which allowed both parents to work uninterrupted wellbeing was higher. Our findings agree with research showing that despite paternal engagement in child and family care rising significantly during the pandemic mothers undertook more childcare (Andrew et al., 2020b; Craig \& Churchill, 2020). Additionally, they are in line with past research highlighting the importance of paternal involvement in childcare for a harmonious stressfree family life (Allport et al., 2018). Although we did not examine specifically if mothers spent more time caring/homeschooling while working compared to fathers, it was reported as one of the most challenging aspects of the lockdown. Additionally, a few mothers expressed resentment and frustration for the unequal distribution of uninterrupted work time. Recent research has shown that mothers spent more time than fathers working while caring for the children and for other tasks which can influence productivity and in turn increase anxiety (Andrew et al., 2020a). Understanding the intersection of maternal wellbeing and paternal involvement during the lockdown can inform the work being undertaken to advise couples about how to work together to adapt to the secondary impacts of the pandemic. Furthermore, our findings contribute to the work that supports a non-gendered approach to the caring role (Vohra \& Taneja, 2020) and its disassociation from traditional notions of masculinity and femininity.

Perceived lack of support from work including lack of flexibility expressed as expectation to be available during normal working hours and increased workload was another important challenge, with lone mothers being severely impacted. Where employers provided more support such as less pressure and more flexibility in the form of not having to be available at the time that home schooling takes place mothers were more likely to report better wellbeing. The above findings suggest that remote working does not serve everyone during the pandemic as it often means working while homeschooling/childcaring. A less rigid approach in conceptualising flexible working would allow consideration of a greater pool of working options that are determined by individual need and could range from less pressure for face time to key worker status for lone mothers when appropriate, as a participant suggested. Also, while some mothers reported that they prefer working from home due to heavier household responsibilities caution is required not perpetuate women's dual role which may contribute to missing career opportunities (Hupkau \& Petrongolo, 2020). 
Regarding school support a few reported dissatisfaction including lack or insufficient on-line teaching and communication between school and family, and a large volume of taught material. For some these were compounded by poor technological support such as inadequate number of devices and/or internet connection, which is a key ingredient for the success of remote learning according to the Education Endowment Foundation (EEF)'s rapid evidence assessment (2020). The lockdown inequalities in education because of differences in parental involvement, access and confidence with home learning has been documented (Williams et al., 2020). Altogether, the findings raise questions about how schools have managed to respond to remote learning and suggest diversification of support driven by pupil and family need, and reaching out to those who are missing out. Additionally, focusing on quality rather than quantity (Education Endowment Foundation, 2020) may contribute to increasing the impact of home learning. Findings from a large Norwegian sample of teacher and families suggest that a shift to creative teaching, including project-based activities, experiments, and use of nature and the outdoors, could enhance the quality of remote learning (Bubb \& Jones, 2020). Our findings add that implementation of quality teaching should consider how working and lone mothers were able to fit it in seeing the difficulties managing synchronous homeschooling and the challenges accessing and using the range of creative activities provided on-line. On a final note, many mothers in the sample were anxious about child socioemotional wellbeing which aligns with concerns over the impact of the lockdown on children's and young people's mental health (Lee, 2020; Morgül et al., 2020). The impetus for attention to child social and emotional wellbeing, and its association with academic outcomes (Jones \& Kahn, 2018), suggests that the aims of education during as well as post crisis may have to be re-examined and school provision could consider integrating academic as well as social and emotional learning goals more systematically in the curriculum.

Finally, another important finding was maternal coping strategies. Overall, mothers who reported more positive experiences were distinguished by a positive mentality characterised by attention to the present, including appreciating the opportunity to bond with the family because of spending more time, together focusing on promoting family and child wellbeing, and gratitude. These attributes reflect skills that are targeted by mindfulness training which focuses on attending in a non-judgmental or accepting way to the present, instead of preoccupying oneself with memories, fantasies, worries, and plans, or responding in a mechanical manner unaware of one's actions (Baer et al., 2004). Mindfulness-based interventions have been found to be useful in improving mental health particularly symptoms of depression (Duarte et al., 2019). Therefore, easy, and timely access to low intensity mindfulness based psychological therapies during and after the pandemic could be a way forward. However, in the UK access to these services has been associated with problems with referrals (Thomas et al., 2020), long waiting lists (Marshall et al., 2016), and mixed views about their effectiveness (Scott, 2019; Wakefield et al., 2020).

The study had some methodological limitations. The sample comprise primarily white mothers with a higher education degree, so caution is required when generalising to the wider population of working mothers. Nevertheless, they provide an informed source of the range and nature of challenges experienced by mothers in similar situations. Because the survey might have been accessed by mothers who were feeling particularly overwhelmed and distressed with the extra responsibilities, maternal 
impressions and emotions could be exaggerated. While we should be cautious extrapolating to the wider population of working mothers, understanding the experiences of those who struggled the most can inform the design of targeted interventions. Our findings were not triangulated with quantitative or multiple methods of data collection but having two coders contributed to enhancing the validity of the analysis and the findings.

\section{Declarations}

Funding: The authors received no funding.

Data availability statement: The data that support the findings of this study are available from the corresponding author, [AK], upon reasonable request.

Conflicts of interest/Competing interests: The authors declare no conflict of interest/competing interests.

Acknowledgements: We are grateful to Venla Panula, University of Turku and Nelli Lyyra, University of Jyväskylä for contributing to the development of the survey on maternal mood during the lockdown 2020. Special thanks also to all the mothers who participated in our survey and for generously sharing their personal experiences during the COVID-19 lockdown in the summer of 2020. We are grateful to them for their openness, honesty, and contribution to the continuous effort to improving children's and families lives during the COVID-19 pandemic.

\section{References}

1. Allport, B. S., Johnson, S., Aqil, A., Labrique, A. B., Nelson, T., Angela, K., Carabas, Y., \& Marcell, A. V. (2018). Promoting father involvement for child and family health. Academic pediatrics, 18(7), 746753.

2. Andrew, A., Cattan, S., Costa Dias, M., Farquharson, C., Kraftman, L., Krutikova, S., Phimister, A., \& Sevilla, A. (2020a). Family time use and home learning during the COVID-19 lockdown. In. London: The Institute for Fiscal Studies.

3. Andrew, A., Cattan, S., Costa Dias, M., Farquharson, C., Kraftman, L., Krutikova, S., Phimister, A., \& Sevilla, A. (2020b). How are mothers and fathers balancing work and family under lockdown. In IFS Briefing Note BN290: Institute for Fiscal Studies.

4. Andrew, A., Cattan, S., Costa Dias, M., Farquharson, C., Kraftman, L., Krutikova, S., Phimister, A., \& Sevilla, A. (2020c). Inequalities in Children's Experiences of Home Learning during the COVID-19 Lockdown in England. Fiscal Studies, 41(3), 653-683.

5. Arnot, J. (2020). COVID-19 and lone

6. parents with dependent children. In. Edinburgh: Public Health Scotland.

7. Baer, R. A., Smith, G. T., \& Allen, K. B. (2004). Assessment of mindfulness by self-report: The Kentucky Inventory of Mindfulness Skills. Assessment, 11(3), 191-206. 
8. Braun, V., \& Clarke, V. (2006). Using thematic analysis in psychology. Qualitative research in psychology, 3(2), 77-101.

9. Bubb, S., \& Jones, M.-A. (2020). Learning from the COVID-19 home-schooling experience: Listening to pupils, parents/carers and teachers. Improving Schools, 23(3), 209-222.

10. Chandola, T., Booker, C. L., Kumari, M., \& Benzeval, M. (2019). Are flexible work arrangements associated with lower levels of chronic stress-related biomarkers? A study of 6025 employees in the UK household longitudinal study. Sociology, 53(4), 779-799.

11. Cooper, K., \& Stewart, K. (2013). Does money affect children's outcomes?

12. Craig, L., \& Churchill, B. (2020). Dual-earner parent couples' work and care during COVID-19. Gender, Work \& Organization.

13. Czymara, C. S., Langenkamp, A., \& Cano, T. (2020). Cause for concerns: gender inequality in experiencing the COVID-19 lockdown in Germany. European Societies, 1-14.

14. Daminger, A. (2019). The cognitive dimension of household labor. American Sociological Review, $84(4), 609-633$.

15. Di Giorgio, E., Di Riso, D., Mioni, G., \& Cellini, N. (2020). The interplay between mothers' and children behavioral and psychological factors during COVID-19: an Italian study. European child \& adolescent psychiatry, 1-12.

16. Duarte, R., Lloyd, A., Kotas, E., Andronis, L., \& White, R. (2019). Are acceptance and mindfulnessbased interventions 'value for money'? Evidence from a systematic literature review. British Journal of Clinical Psychology, 58(2), 187-210.

17. Henderson, A., Harmon, S., \& Newman, H. (2016). The price mothers pay, even when they are not buying it: Mental health consequences of idealized motherhood. Sex Roles, 74(11-12), 512-526.

18. Holmes, E. A., O'Connor, R. C., Perry, V. H., Tracey, I., Wessely, S., Arseneault, L., Ballard, C., Christensen, H., Silver, R. C., \& Everall, I. (2020). Multidisciplinary research priorities for the COVID-19 pandemic: a call for action for mental health science. The Lancet Psychiatry.

19. Hubert, S., \& Aujoulat, I. (2018). Parental burnout: When exhausted mothers open up. Frontiers in psychology, 9, 1021.

20. Hupkau, C., \& Petrongolo, B. (2020). Work, care and gender during the Covid-19 crisis. In.

21. Jones, S. M., \& Kahn, J. (2018). The Evidence Base for How Learning Happens: A Consensus on Social, Emotional, and Academic Development. American Educator, 41(4), 16.

22. Lachance-Grzela, M., McGee, S., \& Ross-Plourde, M. (2019). Division of family labour and perceived unfairness among mothers: the role of mattering to family members. Journal of Family Studies.

23. Lamar, M. R., Forbes, L. K., \& Capasso, L. A. (2019). Helping working mothers face the challenges of an intensive mothering culture. Journal of Mental Health Counseling, 41(3), 203-220.

24. Lee, J. (2020). Mental health effects of school closures during COVID-19. The Lancet Child \& Adolescent Health, 4(6), 421. 
25. Leijdesdorff, S., van Doesum, K., Popma, A., Klaassen, R., \& van Amelsvoort, T. (2017). Prevalence of psychopathology in children of parents with mental illness and/or addiction: an up to date narrative review. Current opinion in psychiatry, 30(4), 312-317.

26. Lively, K. J., Steelman, L. C., \& Powell, B. (2010). Equity, emotion, and household division of labor response. Social Psychology Quarterly, 73(4), 358-379.

27. Lois, J. (2006). Role strain, emotion management, and burnout: Homeschooling mothers' adjustment to the teacher role. Symbolic Interaction, 29(4), 507-529.

28. Mantouvalou, K. (2020). COVID-19 and gender-blind responses: key policies adopted across the UK and EU put many women at risk. British Policy and Politics at LSE.

29. Marshall, D., Quinn, C., Child, S., Shenton, D., Pooler, J., Forber, S., \& Byng, R. (2016). What IAPT services can learn from those who do not attend. Journal of Mental Health, 25(5), 410-415.

30. Meeussen, L., \& Van Laar, C. (2018). Feeling pressure to be a perfect mother relates to parental burnout and career ambitions. Frontiers in psychology, 9, 2113.

31. Morgül, E., Kallitsoglou, A., \& Essau, C. A. E. (2020). Psychological effects of the COVID-19 lockdown on children and families in the UK. Revista de Psicología Clínica con Niños y Adolescentes, 7(3), 4248.

32. Offer, S., \& Schneider, B. (2011). Revisiting the gender gap in time-use patterns: Multitasking and wellbeing among mothers and fathers in dual-earner families. American Sociological Review, 76(6), 809833.

33. Office for National Statistics. (2019). Families and households in the UK: 2019. In. London: ONS.

34. Office for National Statistics. (2020). Parenting in lockdown: coronavirus and the effects on work-life balance. In. London: ONS.

35. Power, K. (2020). The COVID-19 pandemic has increased the care burden of women and families. Sustainability: Science, Practice and Policy, 16(1), 67-73.

36. Scott, M. J. (2019). Ensuring IAPT Makes A Real-World

37. Difference. Mental Health and Family Medicine, 15, 893-895.

38. Spinelli, M., Lionetti, F., Pastore, M., \& Fasolo, M. (2020). Parents' stress and children's psychological problems in families facing the COVID-19 outbreak in Italy. Frontiers in Psychology, 11, 1713.

39. Sánchez-Rodríguez, R., Orsini, É., Laflaquière, E., Callahan, S., \& Séjourné, N. (2019). Depression, anxiety, and guilt in mothers with burnout of preschool and school-aged children: Insight from a cluster analysis. Journal of affective disorders, 259, 244-250.

40. Thomas, F., Hansford, L., Ford, J., Wyatt, K., McCabe, R., \& Byng, R. (2020). How accessible and acceptable are current GP referral mechanisms for IAPT for low-income patients? Lay and primary care perspectives. Journal of Mental Health, 29(6), 706-711.

41. Topalli, P.-Z., Panula, V., \& Lyyra, N. (2020). Pandemic Mood

42. Questionnaire. 
43. Vohra, S., \& Taneja, M. (2020). Care and community revalued during the COVID-19 pandemic: A feminist couple perspective. Gender, Work and Organization.

44. Wakefield, S., Kellett, S., Simmonds-Buckley, M., Stockton, D., Bradbury, A., \& Delgadillo, J. (2020). Improving Access to Psychological Therapies (IAPT) in the United Kingdom: A systematic review and meta-analysis of 10-years of practice-based evidence. British Journal of Clinical Psychology, e12259.

45. Wenham, C., Smith, J., \& Morgan, R. (2020). COVID-19: the gendered impacts of the outbreak. The lancet, 395(10227), 846-848.

46. Williams, T., Mayhew, M., \& Lagou, M. (2020). Coronavirus and homeschooling in Great Britain: April to June 2020. In (Vol. 2020): ONS London. 